\title{
Should pulse pressure influence prescribing?
}

\section{Anthony M Dart \\ Professor of Cardiovascular Medicine \\ Department of Medicine \\ Central Clinical School \\ Monash University \\ Director of Cardiovascular Medicine \\ Alfred Health \\ Melbourne}

\section{Keywords}

atherosclerosis, blood pressure, cardiovascular disease

Aust Prescr 2017;40:26-9 http://dx.doi.org/10.18773/ austprescr.2017.006

\section{SUMMARY}

The pulse pressure is the difference between the systolic and diastolic blood pressure. It is influenced by the stroke volume and vascular resistance.

As people age the walls of their arteries become stiffer. This increases the pulse pressure.

A high pulse pressure may be associated with reduced coronary perfusion. It may therefore be a predictor of future cardiovascular events, but this has not been confirmed by meta-analysis.

There are no drugs specifically aimed at reducing arterial stiffness. Treatment should be aimed at systolic and diastolic pressure rather than reducing pulse pressure.

\section{Introduction}

Cardiovascular events are more likely in patients with high blood pressure, but low blood pressure may also increase the risk. This J-shaped curve has been seen in some studies of antihypertensive drugs for both systolic and diastolic blood pressures (see Fig.) ${ }^{1,2}$

The pulse pressure is the difference between the systolic and diastolic blood pressure. Classically, a wide (high) pulse pressure is a sign of aortic valve regurgitation and a narrow (low) pulse pressure is a sign of aortic stenosis. In the absence of valvular

\section{Fig. J-curve for diastolic blood pressure}

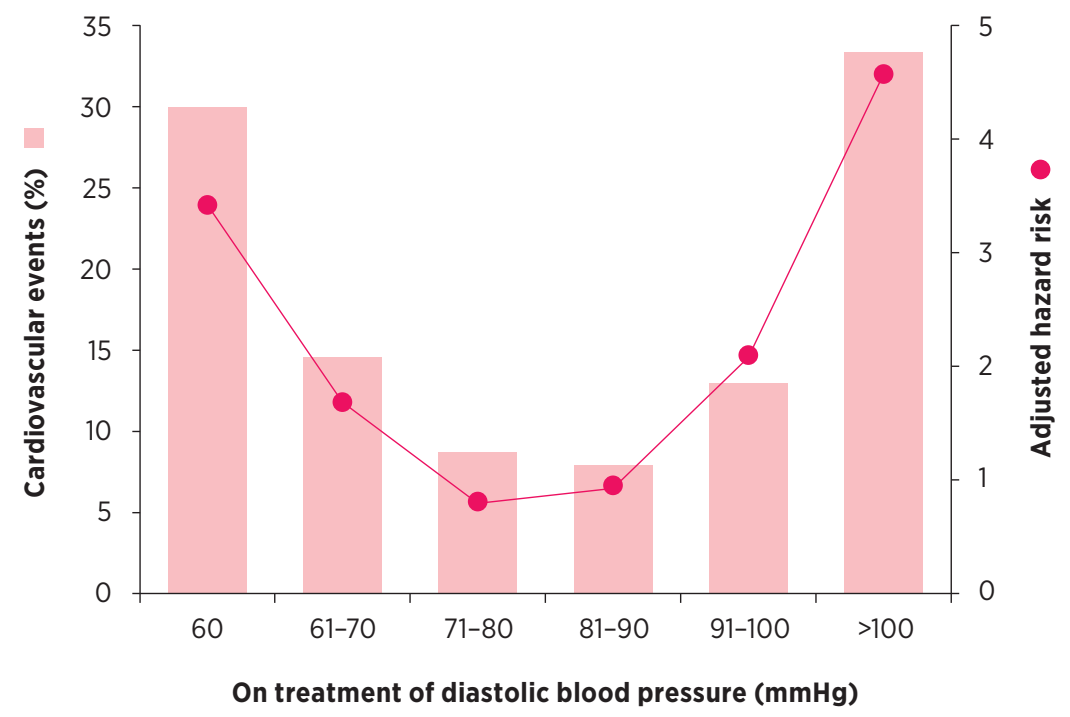

J-curve for patients with coronary artery disease in the Treating to New Targets (TNT) trial $^{1}$

Adapted from Reference 2 disease, a high pulse pressure may be a sign of stiffness in the arterial walls, and is a risk factor for coronary artery disease and myocardial infarction. Many studies have identified pulse pressure as a predictor for future cardiovascular events. In some cases pulse pressure has appeared to be a better predictor than other blood pressure parameters. If pulse pressure is a predictor of mortality, the question arises as to whether altering the pulse pressure will improve clinical outcomes.

\section{Physiology}

The systemic arterial circulation consists of a pulsatile pump, the left ventricle, and a distributive arterial network comprising the aorta, large arteries and the microcirculation. As the pump is pulsatile, a haemodynamic description of the systemic circulation may be considered in terms of mean and pulse pressure. The mean arterial pressure is determined by cardiac output and peripheral vascular resistance, while the pulse pressure is the difference between the systolic and diastolic blood pressures.

The magnitude of the pulsatile component of the systemic arterial pressure largely results from the interaction between left ventricular stroke volume and the compliance of the arterial system, with possibly some additional contribution from wave reflection. Pressure waves travelling from the heart to the periphery may be subject to wave reflection. This is where the forward-travelling pressure wave is reflected back to the heart, particularly at points where the arterial circulation becomes narrowed. The magnitude and clinical significance of wave reflection is a topic of some uncertainty.

The compliance of the arterial circulation is defined as the increase in contained volume in response to pressure increase and results from the fact 
that arteries are distensible. The degree of their distensibility varies throughout the circulation being highest in the proximal aorta which therefore has the greatest compliance in the arterial circulation.

The magnitude of large artery compliance is a function of arterial geometry (mainly diameter) and the properties of the arterial wall, predominantly wall stiffness. In healthy young people the wall is not stiff and therefore 'buffers' each left ventricular ejection. This limits the rise in systolic pressure and provides a supplemental pump to deliver blood flow during diastole. With ageing and certain diseases the large arteries become stiffer and progressively less able to provide the 'buffer' function. This results in a rise in systolic blood pressure and a decrease in diastolic blood pressure therefore widening the pulse pressure.

The acute response of large artery walls to increasing pressure is non-linear. As the blood pressure rises the walls become stiffer. A rise in mean pressure will therefore cause an increase in stiffness and a widening of pulse pressure. From middle age, a rise in pulse pressure is largely dependent on the degree of stiffness of the large arteries whereas in younger people it is largely a function of left ventricular stroke volume.

In addition to ageing, atherosclerosis increases the stiffness of large arteries. Increased arterial stiffness may thus be a surrogate marker for atherosclerotic vascular disease. A widened pulse pressure may be a marker for the extent of coronary disease. Measurement of pulse wave velocity (which increases with increased stiffness) has been proposed as a useful addition to risk assessment. (Pulse wave velocity refers to the transit time of the pressure wave, i.e. energy, and not mass movement of blood.)

\section{Consequences of changing pulse pressure}

The physiological consequences of stiffened large arteries may be related to both the rise in systolic blood pressure and the fall in diastolic blood pressure. The rise in systolic and pulse pressure may lead to further vascular damage and stiffness creating a deleterious feedback loop. ${ }^{3}$ Experimentally enhanced pressure cycles have been shown to lead to accelerated vascular damage raising the possibility of a cyclical cause and effect whereby a stiffened vessel leads to amplified pulse pressure and further vascular damage.

In addition to the vascular consequences of elevated systolic pressure there is an increase in left ventricular afterload which may contribute to impaired left ventricular function. This may eventually result in an impaired capacity to generate the previously maintained stroke volume and hence an adequate pulse pressure. The relation between arterial compliance, stroke volume and pulse pressure is:

$$
\text { pulse pressure }=\frac{\text { stroke volume }}{\text { compliance }}
$$

A fall or rise in stroke volume at a given level of arterial compliance will therefore also affect pulse pressure.

The fall in diastolic pressure seen with pulse pressure widening may be particularly important for coronary perfusion since this occurs predominantly during diastole. In stenotic coronary arteries the reduced diastolic pressure could be expected to lead to impaired myocardial perfusion. Simultaneous measurements of blood pressure and ST segment depression in patients with angina showed a relationship between episodes of 'silent' ischaemia and immediately preceding hypotension noticeable at diastolic blood pressures below $65 \mathrm{mmHg} .{ }^{4}$ This could explain the J-shaped relation between diastolic blood pressure and cardiac events in patients with coronary disease. Reduced perfusion is likely to be more relevant with a shortened diastolic duration (i.e. fast heart rate) and this may indicate the value of choosing therapy that will limit this reduction.

The J-curve is less evident for cerebrovascular disease. This could be because the cerebral vasculature is not dependent on diastolic perfusion.

\section{Therapeutic targets}

The compliant nature of the aorta and large arteries in healthy young people is due to extensive and ordered lamellae of elastin and a high elastin:collagen ratio. With ageing the elastic lamellae become fragmented and disrupted with a proportional increase in (crosslinked) collagen. This leads to a stiffer and less elastic arterial wall. A logical therapeutic strategy would therefore be to try to reverse or at least delay this structural change.

Researchers have studied molecules that would break the collagen cross links and thereby reduce vascular stiffness. The initial results were encouraging, ${ }^{5}$ but the development of an effective drug has not progressed. Of the currently available drugs there was some evidence to suggest that ACE inhibitors and calcium channel blockers may have an effect on stiffness. However, any drug that reduces blood pressure will reduce arterial stiffness due to the non-linear stress-strain relationships of the arterial wall. Attributing changes in arterial compliance to direct effects on the large artery wall is therefore problematic. ${ }^{6}$ 


\section{Blood pressure as a predictor}

The most complete evidence associating blood pressure and mortality comes from a meta-analysis of over one million people from 61 prospective studies. ${ }^{7}$ An important aspect of this analysis was that only patients without known or evident cardiac or cerebrovascular disease were included. This means that it did not include individuals in whom a low diastolic pressure may be harmful. The analysis found that there was a continuous, strong and positive association between both systolic and diastolic blood pressure and cardiovascular events without any apparent lower threshold at least down to a pressure of $115 / 75 \mathrm{mmHg}$. The best predictors of outcome were 'mid' pressure ( $1 / 2$ systolic $+1 / 2$ diastolic) and mean pressure ( $2 / 3$ diastolic $+1 / 3$ systolic). Of the individual components systolic blood pressure was superior to diastolic blood pressure particularly in relation to cardiac disease. In contrast pulse pressure was only about half as predictive as systolic or diastolic pressure. There are no trials in which pulse pressure itself has been either a defined inclusion criterion or a therapeutic target but the prospective data used in the analysis do not suggest that treating pulse pressure, rather than systolic or diastolic blood pressure, in patients with no known cardiac or cerebrovascular events would be a logical approach.

A consequence of increased large artery stiffness is a widened pulse pressure. In such patients reduction of systolic blood pressure is an unambiguous goal. The Systolic Blood Pressure Intervention Trial (SPRINT), ${ }^{8}$ in which patients either had or were at high risk of vascular disease, showed there was evidence that the target systolic blood pressure should be lower than current guidelines suggest. After one year, the intensive treatment group with a mean systolic pressure of $121.4 \mathrm{mmHg}$ had fewer events than the conventional treatment group with a systolic blood pressure of $136.2 \mathrm{mmHg} .{ }^{8}$ In patients with coronary stenoses there is the possibility that a lower diastolic pressure may be associated with more frequent and severe cardiac events. The mean diastolic blood pressure in the intensive arm of the SPRINT was $68.7 \mathrm{mmHg}$.

The relationship between blood pressure and cardiovascular events has also been examined in patients with known coronary artery disease, in the Treating to New Targets (TNT) trial ${ }^{1}$ and International Verapamil-Trandolapril Study (INVEST). ${ }^{9}$ Both trials found evidence of a J-shaped relationship most noticeably for diastolic blood pressure. In the TNT trial the curve relating diastolic blood pressure to events was relatively flat in the range $70-80 \mathrm{mmHg}$ but there was a rise in cardiovascular events in the range $60-70 \mathrm{mmHg}$ (see Fig.). ${ }^{1,2}$ In INVEST a rise in events was evident in patients with systolic blood pressure below $110 \mathrm{mmHg}$ and diastolic blood pressure below $70 \mathrm{mmHg}$. This association was more evident for diastolic blood pressure than systolic blood pressure and with a marked effect if the diastolic blood pressure was below $60 \mathrm{mmHg}$. The HOT study ${ }^{10}$ did not find evidence of a J-shaped relation with diastolic blood pressure in patients with coronary heart disease. However, there was a non-significant rise in cardiovascular mortality below $75 \mathrm{mmHg}$ when the 'optimal' diastolic pressure was in the range 80 to $85 \mathrm{mmHg}$.

There are other possible interpretations of a link between low diastolic blood pressure and cardiovascular events. A low diastolic blood pressure could be a consequence of impaired left ventricular function, however diastolic blood pressure remains a predictor of events after controlling for left ventricular function."

\section{Conclusion}

At present there are no data from randomised clinical trials to support the concept that the reduction of elevated pulse pressure should be a therapeutic goal. While there are some observational data that pulse pressure is a superior predictor of events compared to individual measures of systolic blood pressure and diastolic blood pressure, ${ }^{12,13}$ this is not supported by a large meta-analysis of people with no known cardiovascular events.

There is some evidence, and a plausible mechanistic basis, to suggest that a particularly low diastolic blood pressure is disadvantageous for patients with known coronary artery disease. In this group it would be prudent to avoid inducing excessively low diastolic blood pressure, especially if the patient has a fast heart rate. $<$

Acknowledgement: The author is indebted to Professor James Cameron, Director Monash Cardiovascular Research Centre, for his critical evaluation of the manuscript.

Conflict of interest: none declared 


\section{REFERENCES}

1. Bangalore S, Messerli FH, Wun CC, Zuckerman AL, DeMicco D, Kostis JB, et al.; Treating to New Targets Steering Committee and Investigators. J-curve revisited: an analysis of blood pressure and cardiovascular events in the Treating to New Targets (TNT) Trial. Eur Heart J 2010;31:2897-908. http://dx.doi.org/10.1093/eurheartj/ehq328

2. Banach M, Aronow WS. Blood pressure j-curve: current concepts. Curr Hypertens Rep 2012:14:556-66. http://dx.doi.org/10.1007/s11906-012-0314-3

3. Dart AM, Kingwell BA. Pulse pressure--a review of mechanisms and clinical relevance. J Am Coll Cardio 2001;37:975-84. http://dx.doi.org/10.1016/ S0735-1097(01)01108-1

4. Owens P, O'Brien E. Hypotension in patients with coronary disease: can profound hypotensive events cause myocardial ischaemic events? Heart 1999;82:477-81. http://dx.doi.org/ 10.1136/hrt.82.4.477

5. Kass DA, Shapiro EP, Kawaguchi M, Capriotti AR, Scuteri A deGroof RC, et al. Improved arterial compliance by a novel advanced glycation end-product crosslink breaker. Circulation 2001;104:1464-70. http://dx.doi.org/10.1161/ hc3801.097806

6. Zieman SJ, Melenovsky V, Kass DA. Mechanisms, pathophysiology, and therapy of arterial stiffness. Arterioscler Thromb Vasc Biol 2005;25:932-43. http://dx.doi.org/10.1161/01.ATV.0000160548.78317.29

7. Prospective Studies Collaboration. Age-specific relevance of usual blood pressure to vascular mortality: a meta-analysis of individual data for one million adults in 61 prospective studies. Lancet 2002;360:1903-13. http://dx.doi.org/10.1016/ S0140-6736(02)11911-8
8. SPRINT Research Group. A randomized trial of intensive versus standard blood-pressure control. N Engl J Med 2015;373:2103-16. http://dx.doi.org/10.1056/NEJMoa1511939

9. Pepine CJ, Handberg EM, Cooper-DeHoff RM, Marks RG Kowey P, Messerli FH, et al. A calcium antagonist vs a noncalcium antagonist hypertension treatment strategy for patients with coronary artery disease. The International Verapamil-Trandolapril Study (INVEST): a randomized controlled trial. JAMA 2003;290:2805-16. http://dx.doi.org/ 10.1001/jama.290.21.2805

10. Hansson L, Zanchetti A, Carruthers SG, Dahlöf B, Elmfeldt D Julius S, et al.; HOT Study Group. Effects of intensive bloodpressure lowering and low-dose aspirin in patients with hypertension: principal results of the Hypertension Optimal Treatment (HOT) randomised trial. Lancet 1998;351:1755-62. http://dx.doi.org/10.1016/S0140-6736(98)04311-6

11. Protogerou AD, Safar ME, Iaria P, Safar H, Le Dudal K, Filipovsky J, et al. Diastolic blood pressure and mortality in the elderly with cardiovascular disease. Hypertension 2007;50:172-80. http://dx.doi.org/10.1161/ HYPERTENSIONAHA.107.089797

12. Franklin SS, Larson MG, Khan SA, Wong ND, Leip EP, Kannel WB, et al. Does the relation of blood pressure to coronary heart disease risk change with aging? The Framingham Heart Study. Circulation 2001;103:1245-9. http://dx.doi.org/10.1161/01.CIR.103.9.1245

13. White WB. The systolic blood pressure versus pulse pressure controversy. Am J Cardiol 2001;87:1278-81. http://dx.doi.org/ $10.1016 /$ S0002-9149(01)01519-3 